Textures and Microstructures, Vol. 30, pp. 167-189 Reprints available directly from the publisher Photocopying permitted by license only
(C) 1998 OPA (Overseas Publishers Association) N.V. Published by license under the Gordon and Breach Science Publishers imprint. Printed in India.

\title{
MAGNETIC-COUPLING PROPERTIES IN POLYCRYSTALS
}

\author{
L. FUENTES * \\ Centro de Investigacion en Materiales Avanzados, \\ Complezo Industrial Chihuahua, Miguel de Cervantes 120 Chihuahua, Chih, \\ C.P. 31110, México
}

(Received 5 August 1997)

\begin{abstract}
Quantitative Texture Analysis procedures forcharacterisation of polycrystal pyromagnetic, magnetoelectric and piezomagnetic effects are proposed. The considered phenomena link the thermic, electric and elastic subsystems of a given body with its magnetic subsystem. Brief resumes of required thermodynamic, tensor and texture methods are given. Peculiar behaviour of magnetic (axial-tensor) quantities under colour symmetry operations is described. The use of Neumann's Principle in the investigation of the considered effects is discussed. Surface representation of magnetic-coupling properties is analysed in detail. Colour symmetry, combined with the axial nature of the related tensors, determines the shapes of properties' surfaces. Bunge's spherical harmonics treatment of surface representations is adapted to axial-tensor cases. Computer-generated cases of pyromagnetic, magnetoelectric and piezomagnetic textures are solved.
\end{abstract}

Keywords: Pyromagnetic effect; Magnetoelectric effect; Piezomagnetic effect; Polar and axial tensors; Polycrystal averages

\section{INTRODUCTION}

Theoretical principles for calculation of textured polycrystals' physical properties are known. Bunge's treatises $(1969,1982)$ include clear presentations of the fundamental ideas. Recent publications by Mainprice (1994), Humbert (1991), Diz (1992), Matthies (1994), Imhof (1989), Wright (1994), Zuo (1992) and Raymond (1996a,b) indicate present tendencies and efforts to refine and apply prediction techniques.

*Fax: (5214) 3911 12. E-mail: Ifuentes@yakko.cimav.edu.mx. Visiting researcher from Institute of Cybernetics, Mathematics and Physics, Cuba. 
Magnetic properties have been widely investigated. Also in this area Bunge (1989) has contributed an important synthesis. The last-mentioned paper includes a discussion of polycrystalline magnetostriction, an effect that exemplifies coupling properties. Coupling in this case occurs between the elastic and magnetic sub-systems of the investigated object. From the Tensor Analysis point of view, magnetostriction is represented by a fourth-rank $(r=4)$, polar-character tensor.

As to electric coupling phenomena, Fuentes and Raymond (1995) suggest some attention to be devoted to piezoelectricity, a property that is associated to a polar odd-rank $(r=3)$ tensor. The "odd" component of the texture orientation distribution function (ODF) plays a decisive role in the determination of polycrystalline piezoelectricity. Another odd-rank electric coupling property is pyroelectricity $(r=1)$.

To our knowledge, there is a family of cases that has not received a systematic treatment. These are the polycrystal axial-tensor magneticcoupling properties, including pyromagnetism $(r=1)$, magnetoelectric effect $(r=2)$ and piezomagnetism $(r=3)$. Single-crystal magnetic tensors have been described by Zheludev $(1986,1987)$ and by Litvin (1991, 1994). Experimental magnetic-coupling parameters and other considerations in this field have been contributed by Bouree et al. (1996), Bordin et al. (1995), Prajapati et al. (1995), Aoki et al. (1995), Harshe (1993) and Martin (1992). The purpose of this paper is to discuss in a systematic manner, by making use of Quantitative Texture Analysis (QTA) methods, the mentioned polycrystal effects. Our presentation is self-contained regarding crystal physics, magnetic symmetry and texture concepts and algorithms. We make use of the theory of colour symmetry, as described by Shubnikov and Belov (1964). Application of this theory to QTA was introduced by Bunge et al. (1980). Fuentes and Font (1993) have applied symmetry-antisymmetry formalisms to electric and magnetic fields analyses. Crystal physics background may be found in the International Tables for Crystallography (1996), in Nye's (1957) classic book, in the papers by Fumi and Ripamonti $(1986,1987)$ and in a recent book by Nowick $(1995)$.

\section{CONSTITUTIVE RELATIONS}

We briefly summarise the macroscopic characterisation of reversible thermal, elastic, electric and magnetic interactions. A thermodynamic 
derivation of the following formalisms may be found, for example, in Berlincourt's (1964) review.

Independent variables are selected according to the conditions affecting the considered system. To fix ideas, let us assume that the experimental set-up allows us to control temperature $\theta$, stress $\boldsymbol{T}$, electric field intensity $\boldsymbol{E}$ and magnetic field intensity $\boldsymbol{H}$. We take these quantities as independent variables. Related dependent variables are, in this case, entropy $\sigma$, strain $\boldsymbol{S}$, electric displacement $\boldsymbol{D}$ and magnetic induction $\boldsymbol{B}$.

The behaviour of a given material under the considered actions is described, using a linear approximation, by constitutive relations, Eqs. (1)-(4). Following usual conventions, differential symbols corresponding to mechanical and electromagnetic properties are omitted. Sum over repeated indexes $(i, j, \ldots, n=1,2,3)$ is assumed:

$$
\begin{aligned}
\mathrm{d} \sigma & =\frac{\rho C^{T E H}}{\theta} \mathrm{d} \theta+\alpha_{i j}^{E H} T_{i j}+p_{m}^{T H} E_{m}+i_{n}^{T E} H_{n}, \\
S_{i j} & =\alpha_{i j}^{E H} \mathrm{~d} \theta+s_{i j k l}^{\theta E H} T_{k l}+d_{i j m}^{\theta H} E_{m}+d_{i j n}^{\theta E} H_{n}, \\
D_{m} & =p_{m}^{T H} \mathrm{~d} \theta+d_{m i j}^{\theta H} T_{i j}+\varepsilon_{m n}^{\theta T H} E_{n}+m_{m n}^{\theta T} H_{n}, \\
B_{m} & =i_{m}^{T E} \mathrm{~d} \theta+d_{m i j}^{\theta E} T_{i j}+m_{m n}^{\theta T} E_{n}+\mu_{m n}^{\theta T E} H_{n} .
\end{aligned}
$$

Coefficients in (1)-(4) are material's properties, according to the following definitions, Eqs. (5)-(14). Generally speaking they are tensors. Their ranks $r$ and (polar/axial) characters are included. Supra-indexes denote the independent variables that are held constant during the determination of a given property. $\rho$ is material's density,

$$
\begin{aligned}
\frac{\rho C^{T E H}}{\theta} & \left.=\left(\frac{\partial \sigma}{\partial \theta}\right)_{T E H}, \quad C^{T E H}=\text { heat capacity (scalar, } r=0\right) \\
\alpha_{i j}^{E H} & \left.=\left(\frac{\partial \sigma}{\partial T_{i j}}\right)_{E H}=\left(\frac{\partial S_{i j}}{\partial \theta}\right)_{E H}, \quad \text { thermal expansion (polar, } r=2\right) \\
p_{m}^{T H} & \left.=\left(\frac{\partial \sigma}{\partial E_{m}}\right)_{T H}=\left(\frac{\partial D_{m}}{\partial \theta}\right)_{T H}, \quad \text { pyroelectricity (polar, } r=1\right) \\
i_{m}^{T E} & \left.=\left(\frac{\partial \sigma}{\partial H_{m}}\right)_{T E}=\left(\frac{\partial B_{m}}{\partial \theta}\right)_{T E}, \quad \text { pyromagnetism (axial, } r=1\right) \\
s_{i j k l}^{\theta E H} & =\left(\frac{\partial S_{i j}}{\partial T_{k l}}\right)_{\theta E H}, \quad \text { elasticity }(\text { polar, } r=4)
\end{aligned}
$$




$$
\begin{aligned}
d_{i j k}^{\theta H} & \left.=\left(\frac{\partial S_{i j}}{\partial E_{k}}\right)_{\theta H}=\left(\frac{\partial D_{k}}{\partial T_{i j}}\right)_{\theta H}, \text { piezoelectricity (polar, } r=3\right), \\
d_{i j k}^{\theta E} & \left.=\left(\frac{\partial S_{i j}}{\partial H_{k}}\right)_{\theta E}=\left(\frac{\partial B_{k}}{\partial T_{i j}}\right)_{\theta E}, \text { piezomagnetism (axial, } r=3\right), \\
\varepsilon_{i j}^{\theta T H} & \left.=\left(\frac{\partial D_{i}}{\partial E_{j}}\right)_{\theta T H}, \text { permitivity (polar, } r=2\right), \\
m_{i j}^{\theta T} & \left.=\left(\frac{\partial D_{i}}{\partial H_{j}}\right)_{\theta T}=\left(\frac{\partial B_{j}}{\partial E_{i}}\right)_{\theta T}, \quad \text { magnetoelectricity (axial, } r=2\right), \\
\mu_{i j}^{\theta T E} & =\left(\frac{\partial B_{i}}{\partial H_{j}}\right)_{\theta T E}, \quad \text { permeability }(\text { polar, } r=2) .
\end{aligned}
$$

To schematically describe the considered interactions, we suggest the concentric tetrahedra in Fig. 1. "Effect" magnitudes lie in the inner circles, while "cause" quantities are on outer ones. "Cause-effect" coupling relations are represented by discontinuous lines. Continuous black lines denote "cause-cause" and "effect-effect" links. Broad grey lines are associated to so-called "principal" actions, relating quantities of the same nature. The subject matter of this paper are the coupling properties associated to the magnetic vertex of Fig. 1.

\section{SYMMETRY OF MAGNETIC MAGNITUDES}

We discuss the peculiar behaviour of magnetic quantities under symmetry operations. Already presented $\boldsymbol{B}$ and $\boldsymbol{H}$ fields, as well as magnetisation density $\boldsymbol{M}\left(\boldsymbol{B}=\mu_{0} \boldsymbol{H}+\mu_{0} \boldsymbol{M}\right)$, are typical cases of axial- or pseudo-vectors. They show the interesting property of remaining invariant under coordinates' inversion.

Suitable symmetry concept for investigating materials' magnetic properties is that of colour, complete or generalised symmetry. Let $A(r)$ be a polar vector field and $B(r)$ an axial one. They are both associated to a given object or system. The considered fields satisfy the colour-symmetry operation $G$ if their components fulfil the following conditions:

$$
\begin{aligned}
& A_{i}(G \cdot r)= \pm G_{i j} A_{j}(r), \\
& B_{i}(G \cdot r)= \pm|G| G_{i j} B_{j}(r),
\end{aligned}
$$




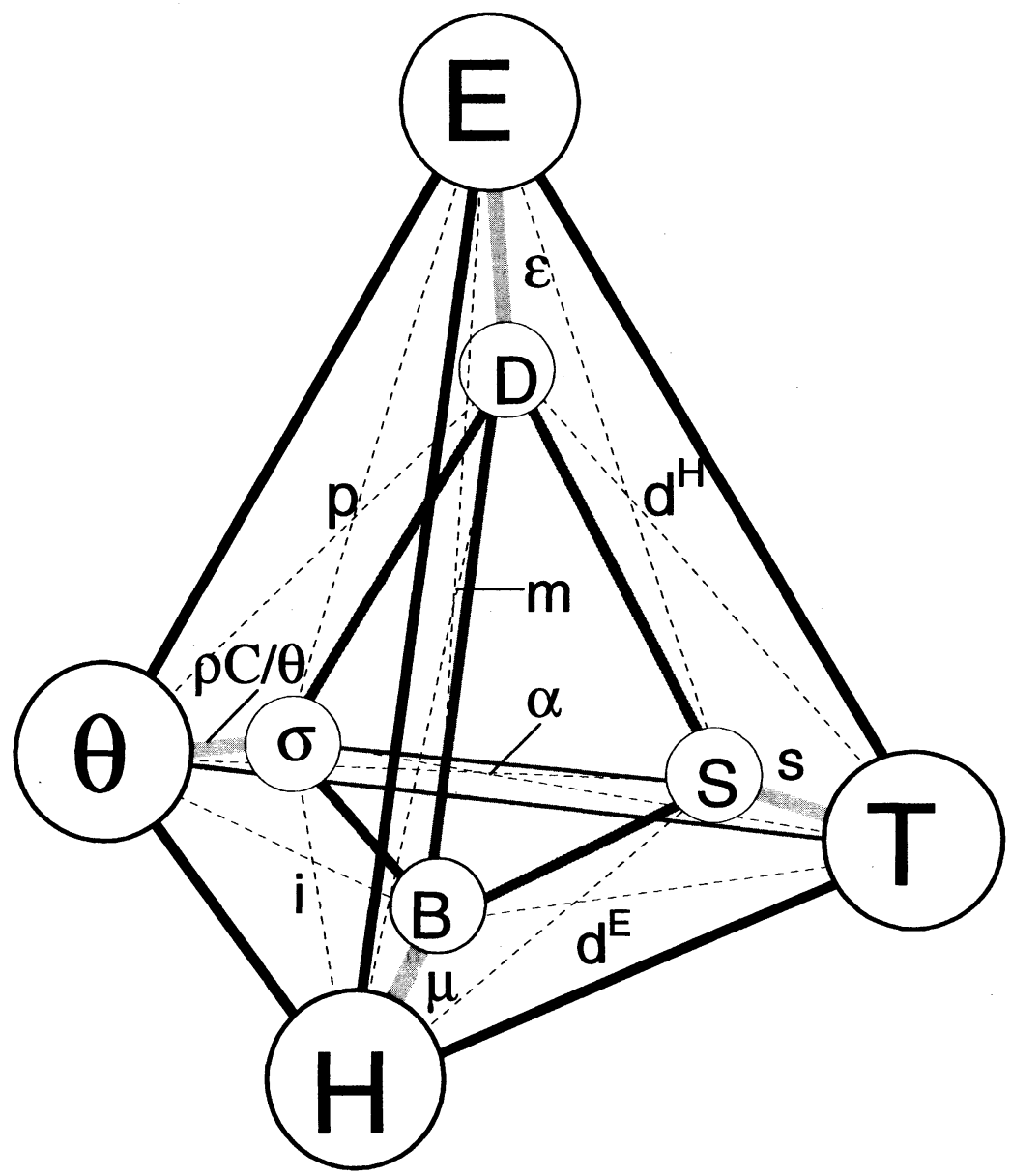

FIGURE 1 Equilibrium properties' tetrahedra.

where $G$ is a proper rotation $(|G|=1)$ or a roto-inversion $(|G|=-1)$. In (15) and (16) plus signs correspond to ordinary symmetry operations while minus signs are associated to anti-symmetry operations. Table I resumes some polar and axial vectors symmetry properties at points lying on symmetry elements.

Consider now the behaviour under symmetry operations of vectors representing physical properties, say pyroelectric and pyromagnetic 
TABLE I Vector conditions at symmetry elements

\begin{tabular}{llcc}
\hline Element & & \multicolumn{2}{c}{ Conditions for } \\
\cline { 3 - 4 } & & $A($ polar $)$ & $B$ (axial) \\
\hline Centre & ordinary inversion & $A=0$ & no conditions \\
& anti-inversion & no conditions & $B=0$ \\
Axis & ordinary rotation & $A \| n$ & $B \| n$ \\
& anti-rotation & $A \perp n^{*}$ & $B \perp n^{*}$ \\
Plane & ordinary mirror & $A \| m$ & $B \perp m$ \\
& anti-mirror & $A \perp m^{*}$ & $B \| m^{*}$ \\
\hline
\end{tabular}

$n \rightarrow$ ordinary-symmetry rotation axis; $n^{*} \rightarrow$ anti-rotation axis; $m \rightarrow$ ordinarymirror plane; $m^{*} \rightarrow$ anti-mirror plane; $\| \rightarrow$ parallel; $\perp \rightarrow$ perpendicular.

constants. The macroscopic conception of a crystal is that of an homogeneous but anisotropic body. Following this idea, colour-symmetry conditions for polar $(\boldsymbol{A})$ and axial $(\boldsymbol{B})$ vectors under transformation $G$ are obtained by application of Eqs. (15)-(16) to points located at the symmetry elements $(G \cdot r=r)$ :

$$
\begin{aligned}
A & = \pm G \cdot A, & A_{i} & = \pm G_{i j} A_{j}, \\
B & = \pm|G| G \cdot B, & B_{i} & = \pm|G| G_{i j} A_{j} .
\end{aligned}
$$

We finally write the algorithms for symmetry operations acting on polar and axial tensors $A_{k l \ldots n}$ and $B_{k l \ldots n}$ representing physical properties of a given object:

$$
\begin{aligned}
& A_{i j \ldots m}= \pm G_{i k} G_{j l} \ldots G_{m n} A_{k l \ldots n}, \\
& B_{i j \ldots m}= \pm|G| G_{i k} G_{j l} \ldots G_{m n} B_{k l \ldots n} .
\end{aligned}
$$

The notation to denote colour-symmetry groups is analogous to that used for ordinary groups, with anti-symmetry operations marked with an asterisk $(*)$.

Figure 2 represents, by means of stereographic projections, symmetry diagrams describing several point groups associated to hexagonal crystals. Directions generated by all colour-symmetry operations are indicated. Polar and axial vectors are considered. Points show orientations towards the North hemisphere while empty circles denote South oriented ones. We shall pay attention to these diagrams in our 


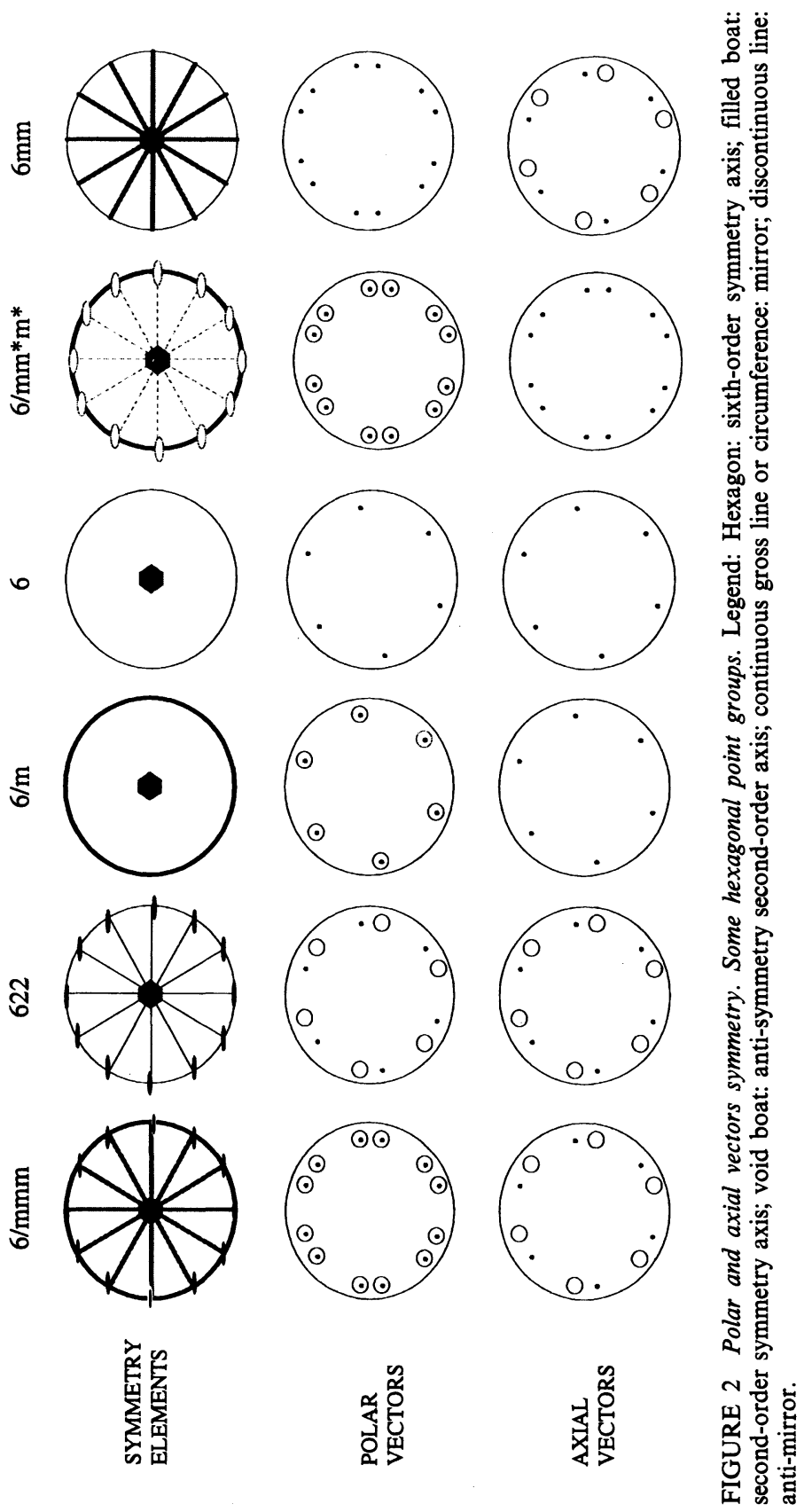


case study of magnetic-coupling in hexagonal crystals and textured polycrystals.

\section{NEUMANN'S PRINCIPLE. SINGLE-CRYSTAL COUPLING EFFECTS}

Pyromagnetic, magnetoelectric and piezomagnetic effects represent coupling properties respectively associated to axial tensors with ranks $r=1,2$ and 3. The defining equations are (8), (13) and (11). Here we rewrite these equations in the so-called matrix notation, Nye (1957). Greek indexes assume the values $\alpha=1,2, \ldots, 6$ :

$$
\mathrm{d} \sigma=i_{m}^{T E} \mathrm{~d} H_{m} ; \quad D_{m}=m_{m n}^{T \theta} H_{n} ; \quad S_{\alpha}=d_{m \alpha}^{E \theta} H_{m} .
$$

Which symmetry operations are satisfied by the physical properties of a given object? A basic idea, known as Neumann's Principle, gives us the answer to this question:

\section{Effect's symmetry is non-less than cause's symmetry.}

Figure 3 exemplifies the application of this principle to magnetism. A hexagonal symmetry uniformly magnetised block is considered. Colour symmetry is $6 / \mathrm{mm}^{*} \mathrm{~m}^{*}$. It would be, say, a cobalt crystal in ferromagnetic state. Notice that conditions (18), (15) and (16) are respectively fulfilled by (uniform) axial vector $M$, polar vector field $J_{M}=\operatorname{rot} M$ and axial vector field $B(r)$. The last magnitude is an "effect", produced by the "cause" $M$ (or its equivalent $J_{M}$ ). One interesting point deserves mention. Our example system is a centrosymmetric one. Nevertheless, if we focus attention on the magnetic field along the $z$ axis, we observe that $B$ always points upwards. The centrosymmetric condition makes up equivalent to down for vectors, but not for pseudo-vectors.

In materials physics, properties are "effects", while "causes" are found in the microscopic structure (the distribution of matter, charge and electric currents). Neumann's Principle for crystal physics may be stated as follows: Generalised-symmetry operations of any physical property conforms a group that contains as a sub-group the coloursymmetry point group of the considered crystal structure.

Mathematically, the tensors of all physical properties satisfy Eqs. (19) or (20), with $G$ scanning all the generalised-symmetry operations of the structural colour point group. 


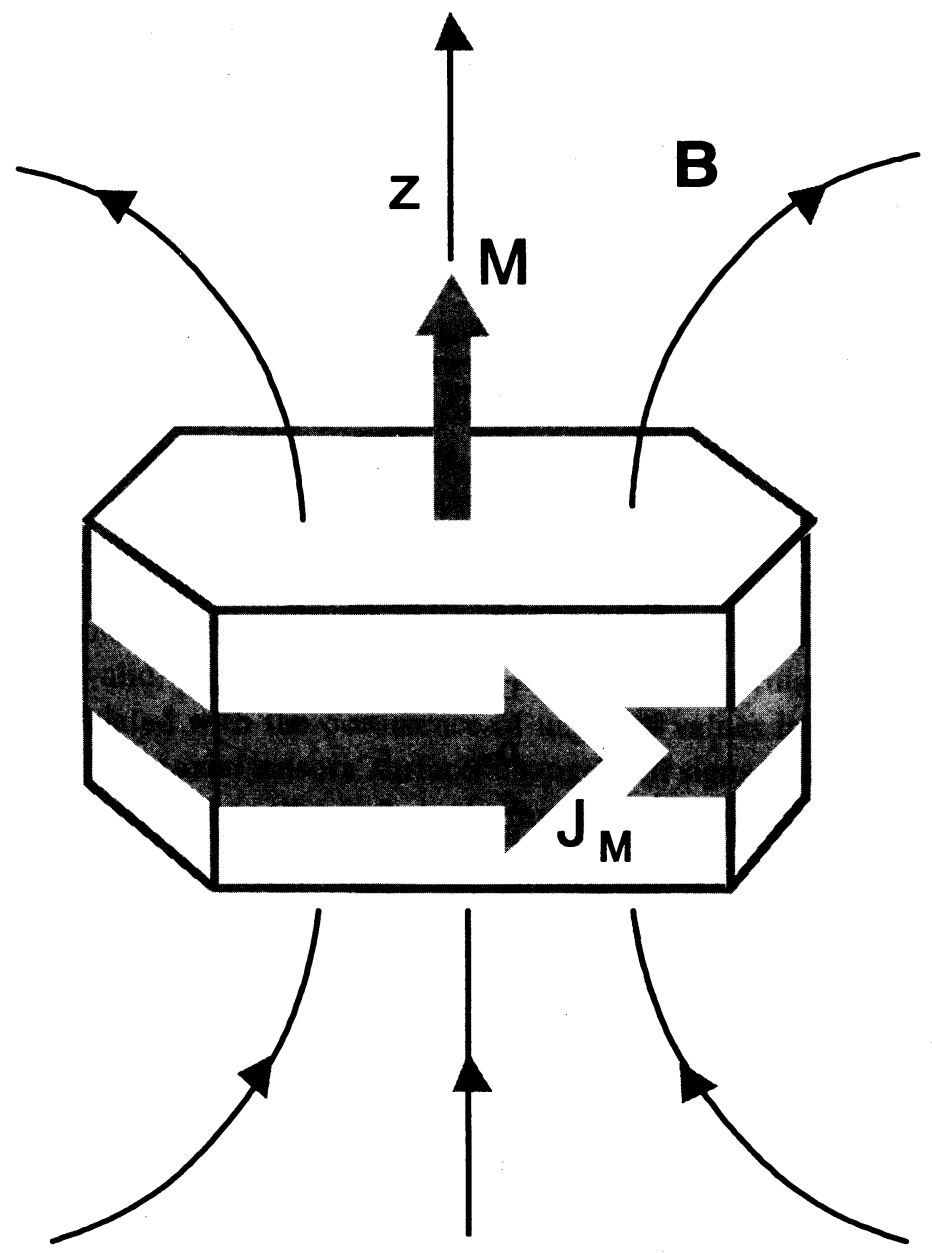

FIGURE 3 Application of Neumann's Principle to a magnetic configuration. Centrosymmetric magnetised hexagon, associated current distribution and magnetic field. Colour-symmetry point group $6 / \mathrm{m}^{*} / \mathrm{m}^{*} 2 * / \mathrm{m}^{*}$.

Let us consider as an example the piezomagnetic tensor of a $6 / \mathrm{mm}^{*} \mathrm{~m}^{*}$ crystal. Conditions (19) lead to matrix expression

$$
d_{i \alpha}^{E \theta}=\left[\begin{array}{cccccc}
0 & 0 & 0 & 0 & d_{15} & 0 \\
0 & 0 & 0 & d_{15} & 0 & 0 \\
d_{31} & d_{31} & d_{33} & 0 & 0 & 0
\end{array}\right]
$$


Components $d_{14}^{E}=-d_{25}^{E}$, while allowed by ordinary-symmetry requirements, are eliminated by anti-symmetry properties.

Table II resumes the possible electric and magnetic-coupling effects in the 32 crystallographic point groups. In electric as well as in magnetic cases, pyro-susceptible materials are a sub-set of piezo-susceptible ones.

TABLE II Electric and magnetic-coupling effects. Crystallographic point groups

\begin{tabular}{|c|c|c|c|c|c|c|c|c|}
\hline \multirow[t]{2}{*}{ Crystallographic system } & \multicolumn{3}{|c|}{ Point group } & \multirow[t]{2}{*}{$P R E$} & \multirow[t]{2}{*}{$P Z E$} & \multirow{2}{*}{\multicolumn{2}{|c|}{ PRM PZM }} & \multirow[t]{2}{*}{$M E$} \\
\hline & Int & Sch & Type & & & & & \\
\hline Triclinic & $\begin{array}{r}1 \\
-1\end{array}$ & $\begin{array}{l}C_{1} \\
C_{i}\end{array}$ & $\begin{array}{l}\mathrm{e} \\
\mathrm{c}\end{array}$ & + & + & $\begin{array}{l}+ \\
+\end{array}$ & $\begin{array}{l}+ \\
+\end{array}$ & + \\
\hline Monoclinic & $\begin{array}{l}2 \\
\mathrm{~m} \\
2 / \mathrm{m}\end{array}$ & $\begin{array}{l}\mathrm{C}_{2} \\
\mathrm{C}_{\mathrm{s}} \\
\mathrm{C}_{2 \mathrm{~h}}\end{array}$ & $\begin{array}{c}\mathrm{e} \\
\mathrm{nc}-\mathrm{ne} \\
\mathrm{c}\end{array}$ & $\begin{array}{l}+ \\
+\end{array}$ & $\begin{array}{l}+ \\
+\end{array}$ & $\begin{array}{l}+ \\
+ \\
+\end{array}$ & $\begin{array}{l}+ \\
+ \\
+\end{array}$ & $\begin{array}{l}+ \\
+\end{array}$ \\
\hline Orthorhombic & $\begin{array}{l}222 \\
2 \mathrm{~mm} \\
\mathrm{mmm}\end{array}$ & $\begin{array}{l}D_{2} \\
C_{2 v} \\
D_{2 h}\end{array}$ & $\begin{array}{c}\text { e } \\
\text { nc-ne } \\
\text { c }\end{array}$ & + & $\begin{array}{l}+ \\
+\end{array}$ & & $\begin{array}{l}+ \\
+ \\
+\end{array}$ & $\begin{array}{l}+ \\
+\end{array}$ \\
\hline Tetragonal & $\begin{array}{c}4 \\
-4 \\
4 / \mathrm{m} \\
422 \\
4 \mathrm{~mm} \\
-42 \mathrm{~m} \\
4 / \mathrm{mmm}\end{array}$ & $\begin{array}{l}C_{4} \\
S_{4} \\
C_{4 h} \\
D_{4} \\
C_{4 v} \\
D_{2 d} \\
D_{4 h}\end{array}$ & $\begin{array}{c}\text { e } \\
\text { nc-ne } \\
\text { c } \\
\text { e } \\
\text { nc-ne } \\
\text { nc-ne } \\
\text { c }\end{array}$ & + & $\begin{array}{l}+ \\
+ \\
+ \\
+ \\
+\end{array}$ & $\begin{array}{l}+ \\
+ \\
+\end{array}$ & $\begin{array}{l}+ \\
+ \\
+ \\
+ \\
+ \\
+ \\
+\end{array}$ & $\begin{array}{l}+ \\
+ \\
+ \\
+ \\
+\end{array}$ \\
\hline Trigonal & $\begin{array}{c}3 \\
-3 \\
32 \\
3 m \\
-3 m\end{array}$ & $\begin{array}{l}C_{3} \\
S_{6} \\
D_{3} \\
C_{3 v} \\
D_{3 d}\end{array}$ & $\begin{array}{c}\mathrm{e} \\
\mathrm{c} \\
\mathrm{e} \\
\mathrm{nc}-\mathrm{ne} \\
\mathrm{c}\end{array}$ & + & $\begin{array}{l}+ \\
+ \\
+\end{array}$ & $\begin{array}{l}+ \\
+\end{array}$ & $\begin{array}{l}+ \\
+ \\
+ \\
+ \\
+\end{array}$ & $\begin{array}{l}+ \\
+ \\
+\end{array}$ \\
\hline Hexagonal & $\begin{array}{l}6 \\
-6 \\
6 / \mathrm{m} \\
622 \\
6 \mathrm{~mm} \\
-6 \mathrm{~m} 2 \\
6 / \mathrm{mmm}\end{array}$ & $\begin{array}{l}C_{6} \\
C_{3 h} \\
C_{6 h} \\
D_{6} \\
C_{6 v} \\
D_{3 h} \\
D_{6 h}\end{array}$ & $\begin{array}{c}\text { e } \\
\text { nc-ne } \\
\text { c } \\
\text { e } \\
\text { nc-ne } \\
\text { nc-ne } \\
\text { c }\end{array}$ & + & $\begin{array}{l}+ \\
+ \\
+ \\
+ \\
+\end{array}$ & $\begin{array}{l}+ \\
+ \\
+\end{array}$ & $\begin{array}{l}+ \\
+ \\
+ \\
+ \\
+ \\
+ \\
+\end{array}$ & $\begin{array}{l}+ \\
+\end{array}$ \\
\hline Cubic & $\begin{array}{c}23 \\
\mathrm{~m} 3 \\
432 \\
-43 \mathrm{~m} \\
\mathrm{~m} 3 \mathrm{~m}\end{array}$ & $\begin{array}{l}T \\
T_{h} \\
O \\
T_{d} \\
O_{h}\end{array}$ & $\begin{array}{c}\mathrm{e} \\
\mathrm{c} \\
\mathrm{e} \\
\mathrm{nc}-\mathrm{ne} \\
\mathrm{c}\end{array}$ & & + & & $\begin{array}{l}+ \\
+\end{array}$ & $\begin{array}{l}+ \\
+\end{array}$ \\
\hline
\end{tabular}

Int: international notation; Sch: Schoenflies notation; e: enantiomorphic; c: centric; nc-ne: noncentric-non-enantiomorphic; +: possible; PRE: pyroelectricity; PZE: piezoelectricity; PRM: pyromagnetism; PZM: piezomagnetism; ME: magnetoelectric effect. 


\section{SURFACE REPRESENTATIONS. THE USE OF SYMMETRY-ADAPTED SPHERICAL HARMONICS}

Let $E_{i j \ldots n}$ be a (polar or axial) tensor property. Its longitudinal surface representation $E(h)$ is given by the algorithm

$$
E(h)=h_{k} h_{j} \cdots h_{n} E_{i j \cdots n},
$$

where $h_{i}$ are the direction cosines of orientation $\mathbf{h}$ with respect to crystallo-physical (orthogonal) axes $O X_{i}$.

The effects of crystal symmetry, with consideration of the polar or axial nature of $E$, are taken into account by means of simplifications on the tensor components $E_{i j \ldots n}$. In crystals that belong to enantiomorphic point groups (see Table II), surfaces associated to axial tensors are equal to those of the corresponding polar tensors. If the inversion symmetry is valid, then some differences appear. A particular interesting point is related with the occurrence of negative values in the longitudinal property: axial tensors' surfaces change their signs under ordinarysymmetry operations that include the inversion symmetry, that is, they behave as pseudo-scalars. If this aspect is taken into account, then the form of the axial longitudinal surface satisfies (Neumann's Principle) point group symmetry. For an axial property, $B(h)$, the analytical generalised expression of this idea is

$$
B(G \cdot h)= \pm|G| B(h),
$$

where $(+)$ and ( $(-)$ signs correspond, respectively, to symmetries and anti-symmetries.

We now present longitudinal surfaces for specific magnetic-coupling properties.

Pyromagnetism: For any pyromagnetic crystal its pyromagnetic tensor is pseudo-vector $i$ pointing along the privileged (say $z$ ) crystal axis. The longitudinal pyromagnetic surface is given by the end points of vector $\boldsymbol{i}$ projections over unitary vectors, $\boldsymbol{h}$, that characterise orientation space. The result is a pair of spheres that are tangent at origin to plane $x y$. A positive sphere lies at the "north" semi-space, while a negative one occupies the "south" semi-space. The equation for $i(h)$ is

$$
i(h)=i_{0} \cos \phi
$$


where $\phi$ is the polar angle, as measured from the privileged axis. The radius of both spheres is $i_{0} / 2$. Surface colour symmetry is given by centrosymmetrical point group $\infty / \mathrm{mm}^{*} \mathrm{~m}^{*}$. Figure 4 includes a representative single-crystal $i(h)$ plot.

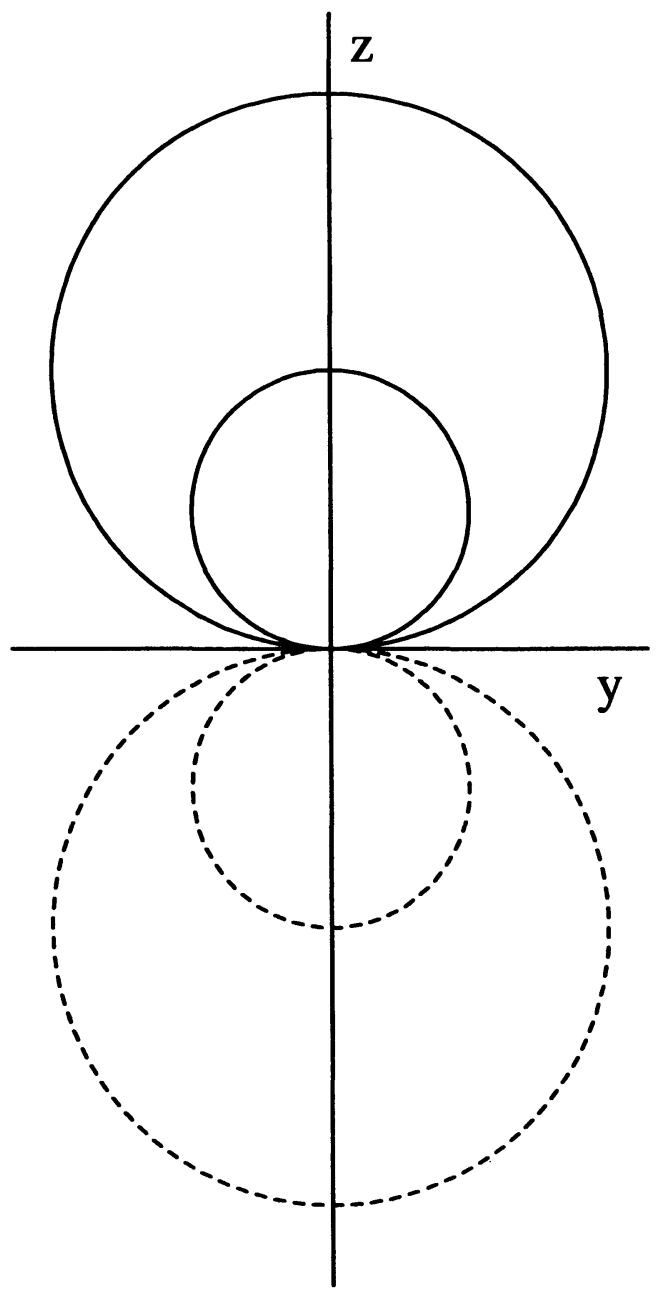

FIGURE 4 Pyromagnetic longitudinal modulus $6 / \mathrm{m} 2 * / \mathrm{m}^{*} 2^{*} / \mathrm{m}^{*}$ crystals, fibre texture. The representative surface is a revolution figure about the $z$ axis. Outer drawings correspond to a single crystal; inner ones to initially untextured magnetised polycrystal. Continuous curves denote positive values of $\langle i\rangle$; discontinuous drawings correspond to negative $\langle i\rangle$. 
Magnetoelectric effect: As in the case of polar second-rank tensors, if crystallo-physical axes are taken along the principal axes of the considered material, the $m(h)$ surface equation is

$$
m(h)=m_{11} h_{1}^{2}+m_{22} h_{2}^{2}+m_{33} h_{3}^{2} .
$$

Depending on crystal symmetry, pseudo-tensor components $m_{i j}$ satisfy conditions that modify $m(h)$ surface's form. Let us consider the case of point group $S_{4}=-4$. By symmetry: $m_{22}=-m_{11}$, with other $m_{i j}=0$. The $m(h)$ surface is

$$
m(h)=m_{11} \sin ^{2} \phi \cos 2 \beta .
$$

Figure 5 includes a cut of the $m(h)$ surface for an $S_{4}$ crystal.

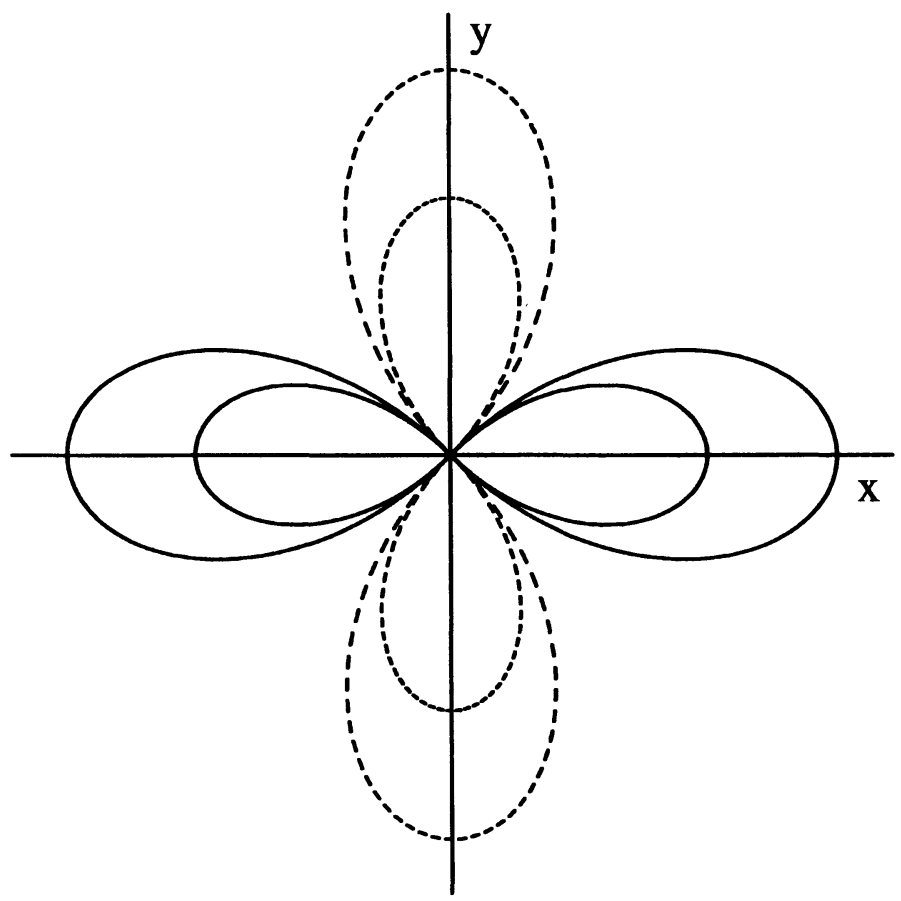

FIGURE 5 Magnetoelectric longitudinal modulus $\mathrm{S}_{4}$ crystals, $\mathrm{C}_{1}$ texture. $\phi=90^{\circ}$ cut. Continuous curves: $m(h)>0$. Discontinuous: $m(h)<0$. Outer drawings: single-crystal. Inner: Gaussian texture, centred at Euler space origin, characteristic width $\Omega_{0}=30^{\circ}$. 
Piezomagnetism: Suitable algorithms are analogous to those given by Fuentes and Raymond (1995) for the piezoelectric case. Axial nature of magnetic magnitudes, however, must be taken into account. Longitudinal piezomagnetic modulus $d^{E}(h)$ is given by

$$
d^{E}(h)=h_{i} h_{j} h_{k} d_{i j k}^{E}
$$

As an example of symmetry-induced simplifications, we write $d^{E}(h)$ for $6 / \mathrm{mm}^{*} \mathrm{~m}^{*}$ colour point group. With tensor's components in matrix notation:

$$
d^{E}(h)=\left(d_{33}-d_{15}-d_{31}\right) \cos ^{3} \phi+\left(d_{15}+d_{31}\right) \cos \phi .
$$

Formula (29) coincides with that of the $6 \mathrm{~mm}$ point group piezoelectric effect (see Fuentes and Raymond, 1995). In Fig. 2, the symmetry diagram of polar vectors for group $6 \mathrm{~mm}$ is the same as that of axial vectors for group $6 / \mathrm{mm}^{*} \mathrm{~m}^{*}$.

We adjust Bunge's $(1969,1982)$ treatment of surface representations to axial-tensors' problems. The general algorithm is condensed in the following equations:

$$
B(h)=\sum_{l=0}^{r} e_{l}^{\mu} k_{l}^{\mu}(h), \quad e_{l}^{\mu}=\int B(h) k_{l}^{\mu} \mathrm{d} h .
$$

The condition to be satisfied by the symmetry-adapted spherical harmonics, $k_{l}^{\mu}(h)$, is

$$
k_{l}^{\mu}(G \cdot h)= \pm|G| k_{l}^{\mu}(h)
$$

where (+) and (-) signs have their usual meanings. Generally speaking, symmetry-adapted functional bases for axial-tensors' surfaces are not the same as those for polar tensors'. For magnetic (axial) surfaces, basic functions are selected among those associated to proper rotations (inversion not included) of the ordinary-symmetry point group.

Let us write magnetic-coupling properties under symmetry-adapted spherical harmonics presentation. We start with pyromagnetism and piezomagnetism. Consider again $6 / \mathrm{mm}^{*} \mathrm{~m}^{*}$ point group. Suitable functional bases are given by triclinic harmonics $K_{1}^{2}(h) ; K_{3}^{4}(h)$ from 
Appendix 1 of Fuentes-Raymond (1995):

$$
\begin{aligned}
& k_{1}^{1}(h)=K_{1}^{2}(h)=\frac{1}{2} \sqrt{\frac{3}{\pi}} \cos \phi=\frac{1}{\sqrt{2 \pi}} P_{1}(\phi), \\
& k_{3}^{1}(h)=K_{3}^{4}(h)=\frac{1}{4} \sqrt{\frac{7}{\pi}}\left(5 \cos ^{3} \phi-3 \cos \phi\right)=\frac{1}{\sqrt{2 \pi}} P_{3}(\phi),
\end{aligned}
$$

where $P_{l}(\phi)$ are Legendre polynomials. Pyromagnetic surface equation becomes

$$
i(h)=e_{1}^{1} k_{1}^{1}(h)=e_{1} P_{1}(\phi)
$$

with

$$
e_{1}^{1}=2 \sqrt{\frac{\pi}{3}} i_{0}=\sqrt{2 \pi} e_{1}
$$

The formula for piezomagnetic $d^{E}(h)$ may be written

$$
d^{E}(h)=e_{1}^{1} k_{1}^{1}(h)+e_{3}^{1} k_{3}^{1}(h)=e_{1} P_{1}(\phi)+e_{3} P_{3}(\phi)
$$

with coefficients

$$
\begin{gathered}
e_{1}^{1}=\frac{2}{5} \sqrt{3 \pi}\left[d_{33}+\frac{2}{3}\left(d_{15}+d_{31}\right)\right]=\sqrt{2 \pi} e_{1}, \\
e_{3}^{1}=\frac{4}{5} \sqrt{\frac{\pi}{7}}\left(d_{33}-d_{15}-d_{31}\right)=\sqrt{2 \pi} e_{3} .
\end{gathered}
$$

Let us now analyse magnetoelectric effect. For $S_{4}$ point group, the associated ordinary-symmetry proper rotations conform to the monoclinic point group $\mathrm{C}_{2}$. Longitudinal modulus $m(h)$ is proportional to "polar" symmetry-adapted harmonic $k_{2}^{3}(h)$ of $\mathrm{C}_{2}$. For reference, triclinic harmonic functions $K_{2}^{\mu}(h)$ are given in Appendix I:

$$
k_{2}^{3}(h)=K_{2}^{5}(h)=\frac{1}{4} \sqrt{\frac{15}{\pi}} \sin ^{2} \phi \cos 2 \beta,
$$


specifically,

$$
m(h)=e_{2}^{3} k_{2}^{3}(h)
$$

with

$$
e_{2}^{3}=4 \sqrt{\frac{\pi}{15}} m_{0}
$$

\section{ALGORITHMS FROM TEXTURE ANALYSIS}

Central magnitudes for description of texture are the orientation distribution functions (ODF). According to the nature of the phenomena under investigation, crystallographic $f(g)$ (Bunge, 1969; 1982), magnetic $f^{[\mathrm{M}]}(g)$ (Bunge, 1989) or electric $f^{[\mathrm{E}]}(g)$ (Fuentes and Raymond, 1995) ODFs are considered. These quantities respectively express the volume fractions $\mathrm{d} \boldsymbol{V}$ of crystallites [domains] in the neighbourhood $\mathrm{d} g$ of the orientation $g=\left(\varphi_{1}, \phi, \varphi_{2}\right) \cdot \varphi_{1}, \phi, \varphi_{2}$ are Euler angles, describing the orientation of crystal [domain] system $K_{B}$ with respect to sample system $K_{\mathrm{A}}$. We shall mostly refer to crystallographic and magnetic structures and magnitudes.

Symmetry plays an important role in texture analysis. The ODF is invariant under proper rotations (inversion not included) that belong to the considered ordinary-symmetry point group. Let the proper rotations from sample symmetry be denoted by $g_{\mathrm{A}_{i}}\left(i=1, \ldots, N_{\mathrm{A}}\right)$ and those from crystal [magnetic] symmetry by $g_{\mathrm{B}_{j}}\left(j=1, \ldots, N_{\mathrm{B}}\right)$ $\left[g_{\mathbf{B}_{j}}^{\mathrm{M}}\left(j=1, \ldots, N_{\mathbf{B}}^{\mathrm{M}}\right]\right.$. Then the following relation holds:

$$
f^{[\mathrm{M}]}(g)=f^{[\mathrm{M}]}\left(g_{\mathrm{A}_{i}} \cdot g \cdot g_{\mathbf{B}_{j}}^{[\mathrm{M}]}\right) .
$$

Three-dimensional spherical harmonics expansion of [magnetic] orientation distribution functions is necessary in the present study. The fundamental equation in this treatment is

$$
f^{[\mathrm{M}]}\left(\varphi_{1}, \phi, \varphi_{2}\right)=\sum_{l=0}^{\infty} \sum_{\mu} \sum_{\nu} C_{l}^{\mu \nu} T_{l}^{\mu \nu}\left(\varphi_{1}, \phi, \varphi_{2}\right),
$$

where $T_{l}^{\mu \nu}(g)$ represent the mentioned harmonics and $C_{l}^{\mu \nu}$ are expansion coefficients. Upper limits for $\mu$ and $\nu$ depend on crystal [magnetic] 
and sample symmetries. Orthogonality conditions for $T_{l}^{\mu \nu}(g)$ allow the calculation of $C_{l}^{\mu \nu}$ on the basis of the knowledge of $f^{[\mathrm{M}]}(\mathrm{g})$ :

$$
C_{l}^{\mu \nu}=(2 l+1) \int f^{[\mathrm{M}]}(g) T_{l}^{\mu \nu} \mathrm{d} g .
$$

Other quantitative descriptions of textures are those given by direct and inverse crystal [magnetic] pole figures. Pole figures are two-dimensional projections of the three-dimensional ODF. If the orientation distribution is symmetric with respect to rotations about a given axis, then we have a fibre texture. In this case the [magnetic] inverse pole figure [M]IPF associated to the symmetry axis $R^{[\mathrm{M}]}(h)$ plays the role of the $[M] O D F$. For this situation, relations (43) and (44) are substituted by

$$
\begin{gathered}
R^{[\mathrm{M}]}(h)=\sum_{l} \sum_{\mu} C_{l}^{\mu} k_{l}^{\mu}(h), \\
C_{l}^{\mu}=\int R^{[\mathrm{M}]}(h) k_{l}^{\mu}(h) \mathrm{d} h .
\end{gathered}
$$

Notice that base $k_{l}^{\mu}(h)$ is symmetric with respect to proper rotations $(|G|=1)$ of the ordinary-symmetry point group. This base contains, as a subgroup, the one associated to the surface representation of axial tensors.

Polycrystal physical properties are texture-modulated manifestations of single-crystal ones. Here we reproduce established algorithms to calculate longitudinal surface representations of polycrystal properties, under the so-called simple average approximation (Bunge, 1982). If single-crystal property and polycrystal texture coefficients $\left(e_{l}^{\mu}\right.$ and $\left.C_{l}^{\mu \nu}\right)$ are known, then polycrystal property is given by

$$
\langle E\rangle=\sum_{l=0}^{r} \sum_{\nu} \xi_{l}^{\nu} k_{l}^{\nu}(y),
$$

where $k_{l}^{\nu}(h)$ are spherical harmonics associated to sample symmetry and the coefficients $\xi_{l}^{\nu}$ are calculated by

$$
\xi_{l}^{\nu}=\frac{1}{2 l+1} \sum_{\mu} C_{l}^{\mu \nu} e_{l}^{\mu}
$$


If the texture shows axial symmetry (fibre texture), Eqs. (47) and (48) are simplified to

$$
\begin{gathered}
\langle E\rangle=\sum_{l=0}^{r} \xi_{l} P_{l}(\phi), \\
\xi_{l}=\frac{1}{4 \pi} \sqrt{\frac{2}{2 l+1}} \sum_{\mu} e_{l}^{\mu} C_{l}^{\mu}
\end{gathered}
$$

with $C_{l}^{\mu}$ calculated according to (46).

\section{PYROMAGNETISM AND PIEZOMAGNETISM IN POLYCRYSTALS}

We investigate pyromagnetic and piezomagnetic effects in polycrystalline materials. To fix ideas, we take a hexagonal material, say cobalt, as model system. We analyse a simple case of fibre texture in detail.

Crystallographic point group of the paramagnetic condition is $6 / \mathrm{mmm}$. Crystal contribution to ODF symmetry is 622 . Symmetry diagrams of both these groups are included in Fig. 2. Magnetisation density, pyromagnetic modulus and piezomagnetic longitudinal modulus are all null under this state. In principle, transverse piezomagnetism is allowed.

Consider now the ferromagnetic state, below the Curie temperature. Apply an intense magnetic field along sample's $z$ axis. After switching off the field, the sample rests in remanence. The ordinary symmetry of the magnetised crystals is now $6 / \mathrm{m}$, colour symmetry is $6 / \mathrm{mm}^{*} \mathrm{~m}^{*}$. The contribution of magnetic-domain symmetry to the symmetry of the magnetic orientation distribution function is given by $C_{6}=6$ point group.

If the sample did not show an initial texture, then the magnetic texture would be axially symmetric (fibre texture) and the resulting magnetisation would point along the $z$ axis.

Let us analyse in detail the initially random case. After magnetisation, the IMPF shows a population $R^{\mathrm{M}}(h)=2$ over the whole northern 
hemisphere, with $R^{\mathrm{M}}(h)=0$ over the southern hemisphere. Texture coefficients for calculation of $\langle i\rangle,\left\langle d^{E}\right\rangle$ are determined as follows:

$$
C_{l}^{1}=2 \pi \int_{0}^{\pi / 2} 2 k_{l}^{1}(h) \sin \phi \mathrm{d} \phi, \quad l=1,3 .
$$

Symmetry-adapted spherical harmonics are those given by Eqs. (32) and (33). Operating

$$
C_{1}^{1}=\sqrt{3 \pi}, \quad C_{3}^{1}=-\frac{\sqrt{7 \pi}}{4} .
$$

Longitudinal moduli are

$$
\begin{gathered}
\langle i\rangle=\frac{i_{0}}{2} \cos \phi, \\
\left\langle d^{E \theta}\right\rangle=\frac{1}{8}\left(d_{31}+d_{15}+d_{33}\right) \cos ^{3} \phi+\frac{1}{8}\left(d_{15}+d_{31}+3 d_{33}\right) \cos \phi .
\end{gathered}
$$

Figure 4 shows a plot of the pyromagnetic case. Single-crystal surface is included.

\section{POLYCRYSTAL MAGNETOELECTRIC EFFECT}

We now discuss the longitudinal magnetoelectric effect in textured polycrystals. Our model system is formed by crystallites with $\mathbf{S}_{\mathbf{4}}$ symmetry. The proper rotations subgroup is monoclinic $\mathrm{C}_{2}\left(N_{\mathrm{B}}=2\right)$. Sample symmetry is triclinic $\mathrm{C}_{1}\left(N_{\mathrm{A}}=1\right)$. Irreducible region in Euler space is $0 \leq \varphi_{1} \leq 2 \pi, 0 \leq \varphi_{2}, \phi \leq \pi$. Our model texture is defined as a Gaussian orientation bell around the origin of Euler space. In the $\phi=0$ plane, all orientations represented by the line $\varphi_{1}+\varphi_{2}=\pi$ show population maxima that are equivalent to that at the origin.

The definition equation for $f(g)$ is

$$
f(g)=f_{0} \sum_{j} \mathrm{e}^{\left(-\Omega_{j} / \Omega_{0}\right)^{2}},
$$

where $\Omega_{j}$ is the angular distance between the orientation $g$ and the considered component maximum $g_{j}^{*}$. $f_{0}$ is such that the usual normalisation condition holds. 
For the considered system, the polycrystal longitudinal magnetoelectric coefficient is

$$
\langle m\rangle=\sum_{\nu=1}^{5} \xi_{2}^{\nu} K_{2}^{\nu}(y)
$$

where $K_{2}^{\nu}(y)$ are triclinic spherical harmonics (Appendix I). Expansion coefficients are given by

$$
\xi_{2}^{\nu}=\frac{1}{5} \sum_{\mu=1}^{5} C_{2}^{\mu \nu} e_{2}^{\mu}=\frac{1}{5} C_{2}^{3 \nu} e_{2}^{3},
$$

where $C_{2}^{3 \nu}$ may be calculated as follows:

$$
C_{2}^{3 \nu}=\frac{5}{S} \int \mathrm{e}^{-\left(\Omega_{l} / \Omega_{0}\right)^{2}} T_{2}^{3 \nu} \mathrm{d} G
$$

Triclinic-monoclinic tridimensional spherical harmonics, for $\mathbf{1}=2$ are calculated following Raymond et al. (1996). Used $T_{2}^{3 \nu}(y)$, are presented in Appendix II. $S$ is given by:

$$
S=\int \mathrm{e}^{-\left(\Omega_{1} / \Omega_{0}\right)^{2}} \mathrm{~d} g .
$$

For the component at origin, the angular distance $\Omega_{1}$ is

$$
\Omega_{1}=A \cos \left\{\frac{1}{2}\left[(1+\cos \phi) \cos \left(\varphi_{1}+\varphi_{2}\right)+\cos \phi-1\right]\right\} .
$$

For the $\Omega_{0} \rightarrow 0$ limiting case, the ODF $f(g)$ tends to a collection of Dirac delta functions $\delta\left(g-g_{j}^{*}\right)$. Naturally, average magnetoelectric modulus coincides with that of a monocrystal.

Consider $\Omega_{0}=30^{\circ}$. Evaluation of (58) by numerical methods, leads to the values shown in Table III. The synthesis of $\langle m\rangle$ drives to the results drawn in Fig. 5 (monocrystal case is included).

TABLE III Expansion coefficients $C_{2}^{3 \nu}$ for Gaussian $\left(\Omega_{0}=30^{\circ}\right)$ texture

\begin{tabular}{lccccc}
\hline$\nu$ & 1 & 2 & 3 & 4 & 5 \\
$C_{2}^{3 \nu}$ & 0.000 & 0.006 & 0.004 & 1.200 & 3.348 \\
\hline
\end{tabular}




\section{APPENDIX I: TRICLINIC BIDIMENSIONAL SPHERICAL}

HARMONICS $(l=2)$

$$
\begin{aligned}
& K_{2}^{1}=\frac{1}{4} \sqrt{\frac{15}{\pi}} \sin ^{2} \phi \sin 2 \beta, \\
& K_{2}^{2}=\frac{1}{2} \sqrt{\frac{15}{\pi}} \cos \phi \sin \phi \sin \beta, \\
& K_{2}^{3}=\frac{1}{4} \sqrt{\frac{5}{\pi}}\left(3 \cos ^{2} \phi-1\right), \\
& K_{2}^{4}=\frac{1}{2} \sqrt{\frac{15}{\pi}} \cos \phi \sin \phi \cos \beta, \\
& K_{2}^{5}=\frac{1}{4} \sqrt{\frac{15}{\pi}} \sin ^{2} \phi \cos 2 \beta .
\end{aligned}
$$

\section{APPENDIX II: SELECTION OF TRIDIMENSIONAL} SYMMETRY-ADAPTED SPHERICAL HARMONICS.

\section{TRICLINIC-MONOCLINIC CASE}

$$
\begin{aligned}
& T_{2}^{31}=\frac{1}{4}\left[(1+\cos \phi)^{2} \sin \left(2 \varphi_{2}+2 \varphi_{1}\right)-(1-\cos \phi)^{2} \sin \left(2 \varphi_{2}-2 \varphi_{1}\right)\right], \\
& T_{2}^{32}=\frac{1}{2}\left[(1-\cos \phi)^{3 / 2}(1+\cos \phi)^{1 / 2} \cos \left(2 \varphi_{2}-\varphi_{1}\right)\right. \\
& \left.-(1+\cos \phi)^{3 / 2}(1-\cos \phi)^{1 / 2} \cos \left(2 \varphi_{2}+\varphi_{1}\right)\right], \\
& T_{2}^{33}=-\frac{\sqrt{3}}{2} \sin ^{2} \phi \cos 2 \varphi_{2}, \\
& T_{2}^{34}=\frac{1}{2}\left[(1+\cos \phi)^{3 / 2}(1-\cos \phi)^{1 / 2} \sin \left(2 \varphi_{2}+\varphi_{1}\right)\right. \\
& \left.+(1-\cos \phi)^{3 / 2}(1+\cos \phi)^{1 / 2} \sin \left(2 \varphi_{2}-\varphi_{1}\right)\right], \\
& T_{2}^{35}=\frac{1}{4}\left[(1+\cos \phi)^{2} \cos \left(2 \varphi_{2}+2 \varphi_{1}\right)+(1-\cos \phi)^{2} \cos \left(2 \varphi_{2}-2 \varphi_{1}\right)\right] .
\end{aligned}
$$




\section{References}

Aoki, Y., Sato, H., Sugawara, H., Svoboda, P., Settai, R., Onuki, Y. and Sugiyama, K. (1995). Anisotropic magnetic and transport properties of $\mathrm{NdCu}_{2}$. J. Magnetism and Magnetic Materials, 150, 151-156.

Berlincourt, D.A., Curran, D.R. and Jaffe, H. (1964). Piezoelectric and piezomagnetic materials and their function in transducers, in Physical Acoustics, Vol. 1, Part A, Ed. Mason, W.P., Academic Press, London.

Bouree, F., Baudour, J.L., Elbadraoui, E., Musso, C., Laurent, C. and Rousset, A. (1996). Crystal and magnetic structure of piezoelectric, ferrimagnetic and magnetoelectric aluminium iron oxide $\mathrm{FeAlO}_{3}$ from neutron powder diffraction. Acta Cryst. $B$, 52, 217-222.

Bunge, H.J. (1969). Mathematische Methoden der Texturanalyse. Akademie Verlag, Berlin.

Bunge, H.J., Esling, C. and Muller, J. (1980). The role of the inversion centre in texture analysis. J. Appl. Cryst., 13, 544-554.

Bordin, G., Buttino, G., Cecchetti, A. and Poppi, M. (1995). Magnetoelastic effects in soft nanocrystalline Fe-based alloys. J. Magnetism and Magnetic Materials, 150, 363-370.

Bunge, H.J. (1982). Texture Analysis in Material Science. Butterworths, London.

Bunge, H.J. (1989). Texture and magnetic properties. Text. and Microstruct., 11, 75-91.

Diz, J. and Humbert, M. (1992). Practical aspects of calculating the elastic properties of polycrystals from the texture according to different models. J. Appl. Cryst., 25, 756.

Fuentes, L. and Font, R. (1993). Simetría y electromagnetismo. Rev. Española de Fsica, 7(2), 49.

Fuentes, L. and Raymond, O. (1995). Texture, piezoelectricity and ferroelectricity. Text. and Microstruct., 23, 221-236.

Fumi, F.G. and Ripamonti, C. (1986). Numerical vector representation of physical properties of crystals. Acta Cryst. A, 42, 293.

Fumi, F.G. and Ripamonti, C. (1987). Non-tensorial arrays for physical properties and the direct-inspection method. Acta Cryst. A, 43, 431.

Harshe, G., Dougherty, J.P. and Newnham, R.E. (1993). Magnetoelectric effect in composite materials. Smart Structures and Materials, SPIE Proceedings, 1919, 224-235.

Humbert, M. and Diz, J. (1991). Some practical features for calculating the polycrystalline elastic properties from texture. J. Appl. Cryst., 24, 978.

Humbert, M., Wagner, F., Philippe, M.J. and Esling, C. (1991). Relation between texture and anisotropic properties: some applications to low symmetry materials. Text. and Microstruct., 14-18, 443-461.

Imhof, J. (1989). On the effective physical properties of polycrystalline materials. Acta Cryst. A, 45, 357.

International Tables for Crystallography (1996). Kluwer Acad. Publ., Dordrecht/Boston/ London.

Litvin, D.B. (1994). Magnetic physical-property tensors. Acta Cryst. A, 50, 406.

Litvin, S.Y. and Litvin, D.B. (1991). Rank 0, 1, 2 and 3 magnetic and non-magnetic physical-property tensors. Acta Cryst. A, 47, 290.

Mainprice, D. and Humbert, M. (1994). Methods of calculating petrophysical properties from lattice preferred orientation data. Surv. Geophys., 15, 575-592.

Martin, R.J. and Noel, J.S. (1992). Dynamic stress measurements using piezomagnetism. Proc. 33rd U. S. Symposium on Rock Mechanics, Ed. by Tillerson and Wawersik, 69-77.

Matthies, S., Humbert, M. and Schuman, C. (1994). On the use of the geometric mean approximation in residual stress analysis. Phys. Status Sol. (B), 186, K41-K44.

Nowick, A.S. (1995). Crystal Properties via Group Theory. Cambridge Univ. Press, Cambridge. 
Nye, J.F. (1957). Physical Properties of Crystals: Their Representation by Tensors and Matrices. Clarendon Press, Oxford.

Prajapati, K., Jenner, A.G. and Greenough, R.D. (1995). Magnetoelastic behaviour of aluminium substituted Terfenol-D at elevated temperatures. IEEE Transactions on Magnetics, 31, 3976-3978.

Raymond, O., Fuentes, L. and Gómez, J.I. (1996a). Surface representation of polycrystal physical properties: All crystal classes, simple average approximation. Text. and Microstruct., 28, 81-92.

Raymond, O., Fuentes, L., Gómez, J.I. (1996b). Computer-oriented real spherical harmonics for texture and properties analyses. Text. and Microstruct., 28, 93-104.

Shubnikov, A.S. and Belov, N.V. (1964). Colored Symmetry. Pergamon Press, London.

Wright, S.I. (1994). Estimation of single-crystal elastic constants from textured polycrystal measurements. J. Appl. Cryst., 27, 794.

Zheludev, I.S. (1987). Fizika kristallov i simmetria. Nauka, Moscow.

Zheludev, I.S. (1986). Space and time inversion in physical crystallography. Acta Cryst. $A, 42,122$.

Zuo, L., Humbert, M. and Esling, C. (1992). Elastic properties of polycrystals in the Voigt-Reuss-Hill approximation. J. Appl. Cryst., 25, 751. 